\title{
ON CRITICAL EXPONENTS FOR THE PUCCI'S EXTREMAL OPERATORS 화
}

\section{SUR LES EXPOSANTS CRITIQUES POUR L'OPÉRATEUR EXTRÉMAL DE PUCCI}

\author{
Patricio L. FELMER *, Alexander QUAAS \\ Departamento de Ingeniería Matemática, and Centro de Modelamiento Matemático, \\ UMR2071 CNRS-UChile Universidad de Chile, Casilla 170 Correo 3, Santiago, Chile \\ Received 15 January 2002, accepted 26 September 2002
}

ABSTRACT. - In this article we study some results on the existence of radially symmetric, non-negative solutions for the nonlinear elliptic equation

$$
\mathcal{M}_{\lambda, \Lambda}^{+}\left(D^{2} u\right)+u^{p}=0 \quad \text { in } \mathbb{R}^{N}
$$

Here $N \geqslant 3, p>1$ and $\mathcal{M}_{\lambda, \Lambda}^{+}$denotes the Pucci's extremal operators with parameters $0<\lambda \leqslant \Lambda$. The goal is to describe the solution set in function of the parameter $p$. We find critical exponents $1<p_{+}^{*}<p_{+}^{p}$ that satisfy: (i) If $1<p<p_{+}^{*}$ then there is no non-trivial radial solution of $(*)$. (ii) If $p=p_{+}^{*}$ then there is a unique fast decaying radial solution of $(*)$. (iii) If $p_{+}^{*}<p \leqslant p_{+}^{p}$ then there is a unique pseudo-slow decaying radial solution to (*). (iv) If $p_{+}^{p}<p$ then there is a unique slow decaying radial solution to $(*)$. Similar results are obtained for the operator $\mathcal{M}_{\bar{\lambda}, \Lambda}^{-}$.

(C) 2003 L'Association Publications de l'Institut Henri Poincaré. Published by Elsevier B.V. All rights reserved

MSC: 35J60; 34B18

Keywords: Critical exponents; Non-divergence form operator; Positive radial solution; Pucci's operator

RÉSUMÉ. - Dans cet article nous avons étudié quelques résultats d'existence des solutions radiales non négatives pour l'équation elliptique non linéaire

$$
\mathcal{M}_{\lambda, \Lambda}^{+}\left(D^{2} u\right)+u^{p}=0, \quad u \geqslant 0 \quad \text { dans } \mathbb{R}^{N} .
$$

Ici $N \geqslant 3, p>1$ et $\mathcal{M}_{\lambda, \Lambda}^{+}$est l'opérateur extrémal de Pucci avec les paramètres $0<\lambda \leqslant \Lambda$. L'objectif est de décrire l'ensemble des solutions en fonction de $p$. On trouve des exposants

\footnotetext{
The authors are partially supported by Fondecyt Lineas Complementarias grant 8000010, and FONDAP Matemáticas Aplicadas, Chile. The second author is also supported by CONICYT Becas de Postgrado.

* Corresponding author.

E-mail addresses: pfelmer@dim.uchile.cl (P.L. Felmer), quaas11@dim.uchile.cl (A. Quaas).
} 
critiques $1<p_{+}^{*}<p_{+}^{p}$ tels que : (i) Si $1<p<p_{+}^{*}$, alors il n'existe pas de solution radiale non triviale de $(*)$. (ii) $\mathrm{Si} p=p_{+}^{*}$, il existe une unique solution radiale de $(*)$ à décroissance rapide. (iii) $\mathrm{Si} p^{*}<p \leqslant p_{+}^{p}$, il existe une unique solution radiale de $(*)$ à décroissance pseudo-lente. (iv) $\mathrm{Si} p_{+}^{p}<p$, il existe une unique solution radiale de $(*)$ à décroissance lente.

Un résultat similaire est obtenu pour l'opérateur $\mathcal{M}_{\lambda, \Lambda}^{-}$.

(C) 2003 L'Association Publications de l'Institut Henri Poincaré. Published by Elsevier B.V. All rights reserved

\section{Introduction}

In this article we are interested in the study of solutions to the nonlinear elliptic equation

$$
\begin{aligned}
& \mathcal{M}_{\lambda, \Lambda}^{ \pm}\left(D^{2} u\right)+u^{p}=0 \quad \text { in } \mathbb{R}^{N}, \\
& u \geqslant 0 \quad \text { in } \mathbb{R}^{N},
\end{aligned}
$$

where $\mathcal{M}_{\lambda, \Lambda}^{ \pm}$denotes the Pucci's extremal operators with parameters $0<\lambda \leqslant \Lambda$ and $N \geqslant 3, p>1$. The Pucci's extremal operators, that play a crucial role in the study of fully nonlinear elliptic equations, are defined as

$$
\begin{aligned}
& \mathcal{M}_{\lambda, \Lambda}^{+}\left(D^{2} u\right)=\Lambda \sum_{e_{i}>0} e_{i}+\lambda \sum_{e_{i}<0} e_{i} \quad \text { and } \\
& \mathcal{M}_{\lambda, \Lambda}^{-}\left(D^{2} u\right)=\lambda \sum_{e_{i}>0} e_{i}+\Lambda \sum_{e_{i}<0} e_{i},
\end{aligned}
$$

where $e_{i}=e_{i}\left(D^{2} u\right), i=1, \ldots, N$, are the eigenvalues of $D^{2} u$. For more details and equivalent definitions see the monograph of Cabré and Caffarelli [1].

When $\lambda=\Lambda=1$ we observe that the operators $\mathcal{M}_{\lambda, \Lambda}^{ \pm}$simply reduce to the Laplace operator, so (1.1) becomes

$$
\begin{aligned}
& \Delta u+u^{p}=0 \quad \text { in } \mathbb{R}^{N}, \\
& u \geqslant 0 \quad \text { in } \mathbb{R}^{N} .
\end{aligned}
$$

This very well known equation has a solution set whose structure strongly depends on the exponent $p$. When $1<p<p_{N}^{*}:=(N+2) /(N-2)$ and $u$ vanishes at infinity, no nontrivial solution to Eq. (1.4) exists, as can be easily proved using the celebrated Pohozaev identity [17]. If $p=p_{N}^{*}$ then it is shown by Caffarelli, Gidas and Spruck in [2] that, up to scaling, Eq. (1.4) possesses exactly one solution. This solution satisfies additionally that $u(|x|)|x|^{N-2} \rightarrow C$ as $|x| \rightarrow \infty$. When $p>p_{N}^{*}$ then Eq. (1.4) admits radial solutions behaving like $C|x|^{-\alpha}$ near infinity, where $\alpha=2 /(p-1)$.

When we consider Eq. (1.4) on a ball, the critical exponent $p_{N}^{*}$ plays a dual role. In fact, the equation

$$
\begin{aligned}
& \Delta u+u^{p}=0 \text { in } B, \\
& u(x)=0 \quad \text { in } \partial B \text { and } u>0 \text { in } B,
\end{aligned}
$$


does not possess solutions if $p \geqslant p_{N}^{*}$ and admits exactly one when $1<p<p_{N}^{*}$. The critical character of $p_{N}^{*}$ is enhanced by the fact that it intervenes in compactness properties of embeddings between Sobolev spaces, a reason for being known as critical Sobolev exponent.

It is interesting to mention that the nonexistence of solutions to (1.4) when $1<p<p_{N}^{*}$ holds even if we do not assume a given behavior at infinity. This result is known as Liouville type theorem and it was proved by Gidas and Spruck [10], see also the proof by Chen and Li [3]. When $1<p \leqslant N /(N-2):=p^{s}$, then a Liouville type theorem is known for supersolutions of (1.4), that is solutions of the inequality

$$
\begin{aligned}
& \Delta u+u^{p} \leqslant 0 \quad \text { in } \mathbb{R}^{N} \\
& u \geqslant 0 \quad \text { in } \mathbb{R}^{N} .
\end{aligned}
$$

Moreover, it is known that this exponent is optimal, in the sense that solutions to (1.4) exist if $p>p^{s}$. This number is called sometimes the second critical exponent for (1.4). See [9].

In a recent paper [6], Cutri and Leoni extended this result for the Pucci's extremal operators. They consider the inequality

$$
\begin{aligned}
& \mathcal{M}_{\lambda, \Lambda}^{ \pm}\left(D^{2} u\right)+u^{p} \leqslant 0 \quad \text { in } \mathbb{R}^{N} \\
& u \geqslant 0 \quad \text { in } \mathbb{R}^{N},
\end{aligned}
$$

and define the dimension-like numbers

$$
\widetilde{N}_{+}=\frac{\lambda}{\Lambda}(N-1)+1 \quad \text { and } \quad \tilde{N}_{-}=\frac{\Lambda}{\lambda}(N-1)+1 .
$$

Then they prove that for $1<p \leqslant p_{+}^{s}:=\widetilde{N}_{+} /\left(\widetilde{N}_{+}-2\right)$ Eq. (1.7), with the operator $\mathcal{M}_{\lambda, \Lambda}^{+}$, has no non-trivial solution. Similarly, if $1<p \leqslant p_{-}^{s}:=\widetilde{N}_{-} /\left(\widetilde{N}_{-}-2\right)$ then Eq. (1.7) with $\mathcal{M}_{\lambda, \Lambda}^{-}$has no non-trivial solution. It is also shown that this second critical exponent is optimal, exhibiting a solution of the inequality when $p>p_{-}^{s}$.

In view of the results for the semilinear Eq. (1.4) that we have discussed above and the new results for inequality (1.7) just mentioned, it is natural to ask about the existence of critical exponents of the Sobolev type for (1.1). In particular it would be interesting to understand the structure of solutions for Eq. (1.1), and the dual Eq. (1.5), in terms for different values of $p>1$. It would also be interesting to prove Liouville type theorems for positive solutions in $\mathbb{R}^{N}$ and to understand the mechanisms for existence of positive solutions in general bounded domains.

It is the purpose of this paper to undertake this problem in the case of radially symmetric solutions. The general problem seems to be too difficult at this point, and we expect that our results will shed some light on it.

Before we state our results we give a definition to classify the possible radial solutions that Eq. (1.1) may have. 
DEFINITION 1.1. - Assume $u$ is a radial solution of (1.1) then we say that:

(i) $u$ is a pseudo-slow decaying solution if there exist constants $0<C_{1}<C_{2}$ such that

$$
C_{1}=\liminf _{r \rightarrow \infty} r^{\alpha} u(r)<\limsup _{r \rightarrow \infty} r^{\alpha} u(r)=C_{2} .
$$

(ii) $u$ is a slow decaying solution if there exists $0<c^{*}$ such that

$$
\lim _{r \rightarrow \infty} r^{\alpha} u(r)=c^{*}
$$

(iii) $u$ is a fast decaying solution if there exists $0<C$ such that

$$
\lim _{r \rightarrow \infty} r^{\widetilde{N}-2} u(r)=C,
$$

where $\widetilde{N}=\widetilde{N}_{+}$or $\widetilde{N}=\widetilde{N}_{-}$, depending if $\mathcal{M}_{\lambda, \Lambda}^{+}$or $\mathcal{M}_{\lambda, \Lambda}^{-}$appears in $(1.1)$.

We will see later that pseudo-slow decaying solutions of (1.1) change infinitely many times its concavity. See the proof of Proposition 3.3.

Remark 1.1. - We observe that the Pucci's extremal operators are positively homogeneous, second order operators. Then we can show that given a solution $u$ of (1.1) and $\gamma>0$, the function $v_{\gamma}$ defined as $v_{\gamma}(x)=\gamma u\left(\gamma^{\alpha^{-1}} x\right)$ is also a solution of (1.1). Consequently, associated to any solution we have a one parameter family of solutions.

Now we are prepared to state our main results describing the critical exponents for Eq. (1.1). We start with the theorem for the operator $\mathcal{M}_{\lambda, \Lambda}^{+}$.

THEOREM 1.1. - Suppose we consider the Pucci's extremal operator $\mathcal{M}_{\lambda, \Lambda}^{+}$in Eq. (1.1). Suppose in addition that $\widetilde{N}_{+}>2$. Then there are critical exponents $1<p_{+}^{s}<$ $p_{+}^{*}<p_{+}^{p}$, with

$$
p_{+}^{s}=\frac{\widetilde{N}_{+}}{\widetilde{N}_{+}-2}, \quad p_{+}^{p}=\frac{\widetilde{N}_{+}+2}{\widetilde{N}_{+}-2}
$$

and

$$
\max \left\{p_{+}^{s}, p_{N}^{*}\right\}<p_{+}^{*}<p_{+}^{p},
$$

that satisfy:

(i) If $1<p<p_{+}^{*}$ then there is no non-trivial radial solution of (1.1).

(ii) If $p=p_{+}^{*}$ then there is a unique fast decaying radial solution of (1.1).

(iii) If $p_{+}^{*}<p \leqslant p_{+}^{p}$ then there is a unique pseudo-slow decaying radial solution to $(1.1)$.

(iv) If $p_{+}^{p}<p$ then there is a unique slow decaying radial solution to (1.1).

In (ii), (iii) and (iv) uniqueness is meant up to scaling, see Remark 1.1.

Remark 1.2. - When $1 \leqslant \widetilde{N}_{+} \leqslant 2$, it was proved in [6] that, independently of the value of $p$, no non-trivial solution of (1.1) with the operator $\mathcal{M}_{\lambda, \Lambda}^{+}$exists.

Regarding the operator $\mathcal{M}_{\lambda, \Lambda}^{-}$we have a slightly different result. 
THEOREM 1.2. - Suppose we consider the Pucci's extremal operator $\mathcal{M}_{\lambda, \Lambda}^{-}$in Eq. (1.1). Then there are critical exponents $1<p_{-}^{s}<p_{-}^{*}<p_{-}^{p}$, with

$$
p_{-}^{s}=\frac{\widetilde{N}_{-}}{\widetilde{N}_{-}-2}
$$

and

$$
\frac{\widetilde{N}_{-}+2}{\widetilde{N}_{-}-2}<p_{-}^{*}<p_{-}^{p}=p_{N}^{*}
$$

that satisfy:

(i) If $1<p<p_{-}^{*}$ then there no non-trivial radial solution to (1.1).

(ii) If $p=p_{-}^{*}$ then there is a unique fast decaying radial solution to (1.1).

(iii) If $p_{-}^{*}<p \leqslant p_{-}^{p}$ then there is a unique radial solution to (1.1), which is a slow decaying or a pseudo-slow decaying solution.

(iv) If $p>p_{-}^{p}$ then there is a unique slow decaying radial solution to (1.1). In (ii), (iii) and (iv) uniqueness is meant up to scaling.

Remark 1.3. - Conclusion (i) in Theorems 1.1 and 1.2 can be interpreted as a Liouville type theorem for radially symmetric solutions of (1.1). Associated to Theorems 1.1 and 1.2 on the critical exponent for the equation in $\mathbb{R}^{N}$ we can prove a result on the existence of radially symmetric positive solutions of Eq. (1.1) in a ball. See Theorem 5.1 in Section 5.

Remark 1.4. - We observe that $\widetilde{N}_{-} \geqslant N \geqslant 3$.

Remark 1.5. - At this point, a further comment on the numbers $p_{+}^{*}$ and $p_{-}^{*}$ is at place. These critical numbers are not known explicitly in terms of the numbers $N, \lambda$ and $\Lambda$. Actually the whole point of this article is to prove their existence and uniqueness.

In the study of Eq. (1.4) a crucial role is played by the Pohozaev identity. Since the Pucci's extremal operators do not have a divergence form, this kind of identity is no longer available, posing a special difficulty to the problem. However, since we consider only radial solutions, the Pucci's operators take a rather simple form, where the concavity of the solution determines its form. This property still allows to use some techniques developed for equations with operators in divergence form.

Our approach consists in a combination of the Emden-Fowler phase plane analysis with the Coffman-Kolodner technique. We start considering the classical Emden-Fowler transformation that allows us to view the problem in the phase plane. With the aid of suitable energy functions we understand much of the behavior of the solutions. Their asymptotic behavior is obtained in some cases using the Poincaré-Bendixon theorem. This phase plane analysis has been used in related problems by Clemons and Jones [4], Kajikiya [12] and Erbe and Tang [8] among many others.

We continue with the use the Coffman-Kolodner technique. Originally introduced by Kolodner [13] and later used by Coffman [5], this technique consists in the study of the solution of an associated initial value problem, obtained differentiating the solution with respect to the initial value. The function so obtained possesses valuable information 
on the problem. This idea has been used by several authors in dealing with uniqueness questions. We cite in particular the work of Kwong [14], Kwong and Zhang [15] and Erbe and Tang [8]. In our case though we do not differentiate with respect to the initial value, which is kept fixed, but with respect to the power $p$. Thus the variation function satisfies a non-homogeneous equation, in contrast with the situations treated earlier.

Our article is organized as follows. In Section 2. we find some preliminary properties of the operators and of the solutions of the associated initial value problem. In Section 3. we study a dynamical system equivalent to the initial value problem, obtained through the Emden-Fowler transformation. We understand the asymptotic behavior of the solutions, especially when $p$ is outside the range defined by the critical Sobolev exponent $p_{N}^{*}$ and the exponent $p_{-}^{*}$ or $p_{+}^{*}$. In Section 4 we analyze the system from the point of view of the Coffman-Kolodner technique. We study the variation of the solution with respect to the exponent $p$. This is a crucial step in obtaining the uniqueness of the critical exponent. Here we use ideas coming from [15] and [8].

\section{Preliminaries}

In this section we introduce some notation and prove some preliminary results. We start with a lemma allowing to compute the Pucci operator in the case of radially symmetric functions.

Lemma 2.1. - Let $\varphi:(0, \infty) \rightarrow \mathbb{R}$ be a $C^{2}$ function. For $x \in \mathbb{R}^{N} \backslash\{0\}$ define $u(x)=$ $\varphi(|x|)$, then the eigenvalues of $D^{2} u$, the Hessian of $u$, are $\varphi^{\prime \prime}(|x|)$, which is simple, and $\varphi^{\prime}(|x|) /|x|$, which has multiplicity $N-1$.

Proof. - A direct computation shows that

$$
D^{2} u(x)=\frac{\varphi^{\prime}(|x|)}{|x|} I+\left[\frac{\varphi^{\prime \prime}(|x|)}{|x|^{2}}-\frac{\varphi^{\prime}(|x|)}{|x|^{3}}\right] X,
$$

where $I$ is the $N \times N$ identity matrix and $X$ is the matrix whose entries are $x_{i} x_{j}$. Hence we have

$$
D^{2} u(x) \frac{x}{|x|}=\varphi^{\prime \prime}(|x|) \frac{x}{|x|} \quad \text { and } \quad D^{2} u(x) \xi=\frac{\varphi^{\prime}(|x|)}{|x|} \xi,
$$

for every vector $\xi$ such that $\xi \cdot x=0$. From here the lemma follows.

For notational convenience, we will consider for the rest of the paper the parameters $\lambda$ and $\Lambda$ to be just positive numbers and define the operator

$$
\mathcal{M}_{\lambda, \Lambda}\left(D^{2} u\right)=\Lambda \sum_{e_{i}>0} e_{i}+\lambda \sum_{e_{i}<0} e_{i}
$$

so that when $\lambda<\Lambda$ we have $\mathcal{M}_{\lambda, \Lambda}^{+}$and when $\lambda>\Lambda$ we have $\mathcal{M}_{\Lambda, \lambda}^{-}$. We will also consider the dimension-like number $\widetilde{N}=\frac{\lambda}{\Lambda}(N-1)+1$, which corresponds to $\widetilde{N}_{+}$or $\widetilde{N}_{-}$depending on the relative size of $\lambda$ and $\Lambda$. This convention will allow to treat, when possible, both operators at once. 
Now we consider the initial value problem

$$
\begin{gathered}
u^{\prime \prime}=M\left(\frac{-\lambda(N-1)}{r} u^{\prime}-u^{p}\right) \quad \text { in }(0,+\infty) \\
u(0)=\gamma, \quad u^{\prime}(0)=0,
\end{gathered}
$$

where $\gamma>0$ and

$$
M(s)= \begin{cases}s / \Lambda & \text { if } s \geqslant 0, \\ s / \lambda & \text { if } s<0 .\end{cases}
$$

We note that Eqs. (2.1)-(2.2) possesses a unique solution, as can be proved following the arguments given by $\mathrm{Ni}$ and Nussbaum in [16]. This solution is of class $C^{2}$ along all $\mathbb{R}_{+}$. We denote this unique solution as $u(r, p, \gamma)$ and we observe that positive solutions of (2.1)-(2.2) decaying to 0 at infinity correspond to radially symmetric solutions of (1.1). The next two lemmas give some general information about solutions of (2.1)-(2.2).

LEMMA 2.2.-The solutions of (2.1)-(2.2) are decreasing, while they remain positive.

Proof. - Let $u$ be a solution of (2.1)-(2.2). Then, by Lemma 2.1 and the definition of the Pucci's operators, $u$ satisfies in a certain interval $\left(0, r_{1}\right) \subset \mathbb{R}_{+}$, the equation

$$
a u^{\prime \prime}+\frac{b(N-1)}{r} u^{\prime}+u^{p}=0,
$$

for certain $a, b>0$. Hence

$$
\left\{u^{\prime} r^{b(N-1) / a}\right\}^{\prime}=-r^{b(N-1) / a} u^{p} \leqslant 0 \quad \text { in }\left(0, r_{1}\right)
$$

Using this and the fact that $u^{\prime}(0)=0$ we have that $u^{\prime}(r)<0$ for all $r \in\left(0, r_{1}\right)$, and by continuity also $u^{\prime}\left(r_{1}\right) \leqslant 0$. Next, the same argument can be applied in a successive way to the intervals to the right of $\left(0, r_{1}\right)$, where $u$ has a $u^{\prime \prime}$ has a fixed sign. Then $u$ is decreasing in every interval $(0, R)$, where it remains positive.

The next lemma gives the scaling property of Eqs. (2.1)-(2.2) and also (1.1), as announced in the introduction.

LEMMA 2.3. - If $u=u\left(r, p, \gamma_{0}\right)$ is a solution of the initial value problem (2.1)-(2.2) then $\gamma u\left(\gamma^{1 / \alpha} r, p, \gamma_{0}\right)=u\left(r, p, \gamma_{0} \gamma\right)$, for all $\gamma_{0}, \gamma>0$.

Proof. - It follows from Lemmas 2.1 and 2.2 that $u$ satisfies

$$
\begin{array}{ll}
\lambda u^{\prime \prime}+\frac{\lambda(N-1)}{r} u^{\prime}+u^{p}=0 & \text { if } u^{\prime \prime}<0 \quad \text { and } \\
\Lambda u^{\prime \prime}+\frac{\lambda(N-1)}{r} u^{\prime}+u^{p}=0 & \text { if } u^{\prime \prime} \geqslant 0 .
\end{array}
$$

From here we find that $u_{\gamma}=\gamma u\left(\gamma^{1 / \alpha} r, p, \gamma_{0}\right)$ satisfies

$$
\frac{1}{\gamma^{1+2 / \alpha}}\left[\lambda u_{\gamma}^{\prime \prime}+\frac{\lambda(N-1)}{r} u_{\gamma}^{\prime}\right]+\frac{1}{\gamma^{p}} u_{\gamma}^{p}=0 \quad \text { if } u_{\gamma}^{\prime \prime}<0,
$$




$$
\frac{1}{\gamma^{1+2 / \alpha}}\left[\Lambda u_{\gamma}^{\prime \prime}+\frac{\lambda(N-1)}{r} u_{\gamma}^{\prime}\right]+\frac{1}{\gamma^{p}} u_{\gamma}^{p}=0 \quad \text { if } u_{\gamma}^{\prime \prime} \geqslant 0 .
$$

Since $p=1+2 / \alpha$ the lemma follows.

In the next definition we classify the exponent $p$ according to the behavior of the solution of the initial value problem (2.1)-(2.2). We define:

$$
\begin{aligned}
& \mathcal{C}=\{p \mid p>1, u(r, p, \gamma) \text { has a finite zero }\} . \\
& \mathcal{P}=\{p \mid p>1, u(r, p, \gamma) \text { is positive and pseudo-slow decaying }\} . \\
& \mathcal{S}=\{p \mid p>1, u(r, p, \gamma) \text { is positive and slow decaying }\} . \\
& \mathcal{F}=\{p \mid p>1, u(r, p, \gamma) \text { is positive and fast decaying }\} .
\end{aligned}
$$

In view of Lemma 2.3, we notice that these sets do not depend on the particular value of $\gamma>0$.

\section{Emden-Fowler analysis}

An important step in the proof of our results is the phase plane analysis we do to the system when we transform it through the classical Emden-Fowler change of variables

$$
x(t)=r^{\alpha} u(r), \quad r=\mathrm{e}^{t} .
$$

With this transformation, the initial value problem (2.1)-(2.2) reduces to the autonomous differential equation

$$
\begin{gathered}
x^{\prime \prime}=-\alpha(\alpha+1) x+(1+2 \alpha) x^{\prime}+M\left(\lambda(N-1)\left(\alpha x-x^{\prime}\right)-x^{p}\right), \\
x(-\infty)=0, \quad x^{\prime}(-\infty)=0 .
\end{gathered}
$$

From now on, this equation will be called the dynamical system associated to (2.1)-(2.2). We note that a change in $\gamma$ in (2.1)-(2.2) becomes a time translation in (3.1). From (3.1) and (2.3) we see that $x$ satisfies

$$
x^{\prime \prime}=-a x^{\prime}+b x-\frac{x^{p}}{\lambda} \quad \text { if } \lambda(N-1)\left(x^{\prime}-\alpha x\right)+x^{p}>0,
$$

and

$$
x^{\prime \prime}=-\tilde{a} x^{\prime}+\tilde{b} x-\frac{x^{p}}{\Lambda} \quad \text { if } \lambda(N-1)\left(x^{\prime}-\alpha x\right)+x^{p} \leqslant 0
$$

where

$$
a=N-2-2 \alpha, \quad b=\alpha(N-2-\alpha), \quad \tilde{a}=\widetilde{N}-2-2 \alpha \quad \text { and } \quad \tilde{b}=\alpha(\widetilde{N}-2-\alpha) .
$$

The right-half plane is thus divided in two regions by the curve

$$
x^{\prime}=\alpha x-\frac{x^{p}}{\lambda(N-1)} \text {. }
$$


For later reference we denote by $R^{+}$the region above (3.4) and by $R^{-}$the region below (3.4).

LEMMA 3.1. - Let $\left(x(t), x^{\prime}(t)\right)$ be a trajectory of (3.1). Then there is $t_{0}$ such that the trajectory belongs to $R^{+}$for all $t \leqslant t_{0}$, it crosses curve (3.4) transversally and at most once before reaching $x^{\prime}=0$

Proof. - If $y(x)=x(t)$ and $(x, y)$ is a point where the trajectory $\left(x(t), x^{\prime}(t)\right)$ crosses (3.4), we have from (3.1) that

$$
\frac{\mathrm{d} y}{\mathrm{~d} x}=(1+2 \alpha)-\alpha(\alpha+1) \frac{x}{y} .
$$

On the other hand, defining $z=y / x$ and using (3.4), we have that the slope of (3.4) at that point is

$$
m=\alpha-\frac{p x^{p-1}}{\lambda(N-1)}=-2+p z
$$

Then, at the point of intersection we have

$$
\Delta \equiv \frac{\mathrm{d} y}{\mathrm{~d} x}-m<0 \quad \text { if and only if } \quad p z^{2}-(3+2 \alpha) z+\alpha(\alpha+1)>0 .
$$

Solving the quadratic equation we find that $\Delta<0$ if and only if $z \notin[\alpha(\alpha+1) /(\alpha+$ 2), $\alpha$ ]. Using Eq. (3.4) we find that if the trajectory crosses (3.4) with $x^{p-1}<x_{1}^{p-1}=$ $\lambda(N-1) / p$ then it crosses from $R^{-}$to $R^{+}$. From here we see that the trajectory stays in $R^{-}$for $t \leqslant \bar{t}$ for some $\bar{t}$. Thus, in terms of the function $u$ we find then that $u^{\prime \prime}(r)>0$ for $r$ close to 0 , contradicting Lemma 2.2. This proves the first part of the statement.

Using Eq. (3.4) again we find that the crossing is transversal and from $R^{+}$to $R^{-}$, if it occurs when $x^{p-1}>x_{1}^{p-1}=\lambda(N-1) / p$.

Thus, in order to complete the proof we only need to show that the crossing does not occur at $x_{1}$. Since at this point we have $\Delta=0$, to prove this we analyze the second derivatives.

Differentiating (3.4) and evaluating at $x_{1}$ we find

$$
\frac{\mathrm{d}^{2} y}{\mathrm{~d} x^{2}}=-\frac{p-1}{x_{1}}
$$

On the other hand, differentiating (3.1) we find

$$
\left(\frac{\mathrm{d} y}{\mathrm{~d} x}\right)^{2}+y \frac{\mathrm{d}^{2} y}{\mathrm{~d} x^{2}}=-\alpha(\alpha+1)+(1+2 \alpha) \frac{\mathrm{d} y}{\mathrm{~d} x}+\frac{1}{\lambda}\left[\lambda(N-1)\left(\alpha-\frac{\mathrm{d} y}{\mathrm{~d} x}\right)-p x^{p-1}\right],
$$

where we assume that the trajectory stays in $R^{+}$. This is the case when $x<x_{1}$. Evaluating at $x=x_{1}$ and noting that at this point $\mathrm{d} y / \mathrm{d} x=\alpha-1$, we find that

$$
\frac{\mathrm{d}^{2} y}{\mathrm{~d} x^{2}}=-\frac{2}{x_{1}(\alpha-1 / p)} .
$$


But, since $p>1$ we find that

$$
\frac{p-1}{x_{1}}<\frac{2}{x_{1}(\alpha-1 / p)}
$$

which contradicts the fact that the trajectory stays in $R^{+}$for $x<x_{1}$.

The next three propositions deal with the solutions of (1.1) when $p$ stays outside the range between $p_{N}^{*}$ and $p_{+}^{*}$ or $p_{-}^{*}$.

Proposition 3.1. - If

$$
p>\max \left\{\frac{\widetilde{N}+2}{\widetilde{N}-2}, \frac{N+2}{N-2}\right\}
$$

then $p \in \mathcal{S}$, that is, (1.1) possesses a slow decaying solution.

Proof. - First we claim that, while the trajectory $\left(x(t), x^{\prime}(t)\right)$ remains in the first quadrant, $x(t) \leqslant \bar{x} \equiv(\lambda \alpha(N-1))^{1 /(p-1)}$. To prove this claim we define the energy-like function

$$
e(t)=\frac{\left(x^{\prime}\right)^{2}}{2}+\frac{\alpha x^{p+1}}{2 \lambda(N-1)}-\frac{(\alpha x)^{2}}{2} .
$$

Given $\left(x(t), x^{\prime}(t)\right) \in R^{+}$we have that

$$
e^{\prime}(t)=x^{\prime}\left\{-a x^{\prime}+\left(b-\alpha^{2}\right) x+\left(\frac{\alpha(p+1)-2(N-1)}{2 \lambda(N-1)}\right) x^{p}\right\}
$$

from where it follows that $e^{\prime}(t)<0$ when $\left(x(t), x^{\prime}(t)\right) \in R^{+}$. In fact, the curve

$$
x^{\prime}=\alpha x+\frac{\alpha(p+1)-2(N-1)}{2 a \lambda(N-1)} x^{p},
$$

which corresponds to $e^{\prime}=0$, is below the curve (3.4), because

$$
\frac{\alpha(p+1)-2(N-1)}{2 a \lambda(N-1)}=\frac{-(N-2-\alpha)}{\lambda(N-1) a}<\frac{-1}{\lambda(N-1)} .
$$

On the other hand, we have that the points $(0,0)$ and $(x, 0)$, for $x \geqslant \bar{x}$, have energy $e$ greater than or equal to zero. Thus the trajectory crosses (3.4), entering into $R^{-}$in the first quadrant. By Lemma 3.1 the claim follows. Before continuing we observe that when the trajectory crosses the $x$-axis, say at $\left(x_{0}, 0\right) \in R^{-}$, it turns back because after this point $x^{\prime}$ becomes negative. Consequently the whole trajectory remains to the left of $x=\bar{x}$, at least while it stays on the right-half plane.

From the hypothesis on $p$ we have that $a>0$ and $\tilde{a}>0$. Let us define a second energy-like function

$$
E(t)=\frac{\left(x^{\prime}\right)^{2}}{2}+\frac{x^{p+1}}{\Lambda(p+1)}-\frac{\tilde{b} x^{2}}{2}
$$


We have that

$$
E^{\prime}(t)= \begin{cases}x^{\prime}\left\{-a x^{\prime}+(b-\tilde{b}) x+\frac{\lambda-\Lambda}{\lambda \Lambda} x^{p}\right\} & \text { if }\left(x, x^{\prime}\right) \in R^{+}, \\ -\tilde{a}\left(x^{\prime}\right)^{2} & \text { if }\left(x, x^{\prime}\right) \in R^{-}\end{cases}
$$

Call $E^{+}$the region in the phase plane above the curve

$$
x^{\prime}=\frac{\alpha(N-\tilde{N})}{a} x+\frac{\lambda-\Lambda}{a \lambda \Lambda} x^{p}
$$

and $E^{-}$the region below the curve. We have that the curve (3.9) is below the curve (3.4) for $x \in(0, \bar{x})$. Observe that $\bar{x}$ is such that $(\bar{x}, 0)$ is on the intersection of the curves (3.4) and (3.9). Consequently $E^{\prime}<0$ while the trajectory stays in $R^{+}$and when it enters $R^{-}$, that is along the whole trajectory.

From here we see that the trajectory remains inside the bounded region defined by $E=0$. Moreover, it cannot approach a periodic orbit, so that by the Poincaré-Bendixon Theorem (see e.g. [11]), $\left(x(t), x^{\prime}(t)\right) \rightarrow\left(c^{*}, 0\right)$, as $t \rightarrow \infty$, where $c^{*}=(\Lambda \tilde{b})^{1 /(p-1)}$. Note that $\left(c^{*}, 0\right)$ is the unique equilibrium of the dynamical system in the right-half plane. Hence $p \in \mathcal{S}$.

Lemma 3.2. - Let $\left(x(t), x^{\prime}(t)\right)$ be a trajectory to (3.1), then, while $x^{\prime}(t)>0$

$$
x^{\prime} \leqslant \alpha x-\frac{x^{p}}{\lambda N}
$$

Proof. - From (2.1) we have

$$
-\left\{u^{\prime} r^{N-1}\right\}^{\prime}=\frac{u^{p} r^{N-1}}{\lambda} \text { in }\left(0, r_{0}\right)
$$

where $r_{0}$ is the first point such that $u^{\prime \prime}\left(r_{0}\right)=0$. Integrating by parts we get

$$
-u^{\prime}(r) r^{N-1}=\frac{u^{p} r^{N}}{N \lambda}-\int_{0}^{r} p u^{p-1}(s) u^{\prime}(s) \frac{s^{N}}{N \lambda} \mathrm{d} s, \quad \text { for } r \leqslant r_{0},
$$

and then

$$
-u^{\prime}(r) r^{N-1} \geqslant \frac{u^{p} r^{N}}{N \lambda}, \quad \text { for } r \leqslant r_{0} .
$$

Writing this in terms of the dynamical system and using Lemma 3.1 we conclude.

PROPOSITION 3.2. - If

$$
p \leqslant \max \left\{\frac{\widetilde{N}}{\widetilde{N}-2}, \min \left\{\frac{\widetilde{N}+2}{\widetilde{N}-2}, \frac{N+2}{N-2}\right\}\right\}
$$

then $p \in \mathcal{C}$, that is, there is no radial solution to (1.1). 
Proof. - If $\widetilde{N} /(\widetilde{N}-2) \geqslant(N+2) /(N-2)$ the result is a consequence of the work of Cutri and Leoni [6].

To treat the other case, let us assume that the trajectory stays in the right-half plane for all $t \in \mathbb{R}$. Since the curve (3.9) is below the curve (3.4) in the first quadrant and to the left of the line $x=\bar{x}$, and because $a<0$ and $\tilde{a}<0$, we obtain that $E^{\prime}>0$ for all points $\left(x, x^{\prime}\right)$ with $0<x<\bar{x}$.

On the other hand, as $(p+1) \Lambda \tilde{b} \leqslant 2 \lambda \alpha(N-1)$, the closed curve $E=0$ is on the region $0<x \leqslant \bar{x}$, except for the origin. Thus, the trajectory stays outside the bounded region $E<0$. From here we see that it cannot converge to a stationary point because the stationary point has negative energy $E$. It cannot converge to a periodic orbit either because in this case the trajectory should go through the region where $x^{\prime \prime}>0$ and then $E<0$.

To get a contradiction we prove finally that the trajectory is bounded. In fact, from Lemma 3.2 we have that $x$ is bounded and that $x^{\prime}$ is bounded from above. If we assume for that $x^{\prime}(t) \rightarrow-\infty$ then by monotonicity $x$ converges to $x_{0} \geqslant 0$, but this would imply $x^{\prime}(t) \rightarrow 0$.

Thus, we conclude that the trajectory must leave the fourth quadrant.

In the case when $\lambda<\Lambda$, that is for the operator $\mathcal{M}_{\lambda, \Lambda}^{+}$, we have the following result on the existence of pseudo-slow decaying solutions.

PROPOSITION 3.3. - If $\lambda<\Lambda$, then the following statements hold:

(i) $(\widetilde{N}+2) /(\widetilde{N}-2) \in \mathcal{P}$,

(ii) $\mathcal{P} \cap(0,(\widetilde{N}+2) /(\widetilde{N}-2))$ is open, and

(iii) If $p \leqslant(\widetilde{N}+2) /(\widetilde{N}-2)$, then $p \notin \mathcal{S}$.

Proof. - (i) First we observe that if $p=(\widetilde{N}+2) /(\widetilde{N}-2)$ then we have

$$
E^{\prime}(t) \begin{cases}<0 & \text { if }\left(x, x^{\prime}\right) \in R^{+}, \\ =0 & \text { if }\left(x, x^{\prime}\right) \in R^{-}\end{cases}
$$

Then the dynamical system has a family of periodic orbits around the point $\left(c^{*}, 0\right)$. The maximal periodic orbit is tangent to the curve (3.4), let us call this periodic orbit $P$. Then, by the Poincaré-Bendixon Theorem we have that $x$ converge (w-limit) to $P$. Thus we find

$$
\begin{aligned}
& \liminf _{t \rightarrow \infty} x(t)=C_{1}:=\inf \left\{x \mid\left(x, x^{\prime}\right) \in P\right\} \quad \text { and } \\
& \limsup _{t \rightarrow \infty} x(t)=C_{2}:=\sup \left\{x \mid\left(x, x^{\prime}\right) \in P\right\} .
\end{aligned}
$$

We notice that the trajectory $\left(x(t), x^{\prime}(t)\right)$ crosses infinitely many times the curve (3.4), hence the function $u$ changes its concavity infinitely many times.

(ii) If $p^{*} \in \mathcal{P}$ then the trajectory $\left(x(t), x^{\prime}(t)\right)$ crosses infinitely many times the line $x^{\prime}=0$, in particular it crosses two times. Then, as a consequence of the continuous dependence on the initial values for ordinary differential equations, for $p$ close to $p^{*}$ the trajectory crosses also two times the line $x^{\prime}=0$ and, as a consequence, $\left(x(t), x^{\prime}(t)\right)$ is bounded. Using the Poincare-Bendixson Theorem, we conclude then that the w-limit 
of the trajectory is a periodic orbit, since the unique stationary point $\left(c^{*}, 0\right) \in R^{+}$has a neighborhood where the energy is strictly increasing. From here (ii) follows.

If we look at the energy along this periodic orbit we see that this orbit must cross the curve (3.4). Thus the corresponding solution $u$ changes infinitely many times its concavity.

(iii) If the trajectory, with $p \leqslant(\widetilde{N}+2) /(\widetilde{N}-2)$, crosses two times the line $x^{\prime}=0$, then by the argument given in (ii) $p \in \mathcal{P}$. In any other case $p \in \mathcal{C} \cup \mathcal{F}$.

Remark 3.1. - As a consequence of the continuous dependence on the initial value for ordinary differential equation, $\mathcal{C}$ is open.

Remark 3.2. - In the range of $p$ where the solution is pseudo-slow decaying, the periodic orbit of the dynamical system corresponds to a singular solution to

$$
\mathcal{M}_{\lambda, \Lambda}^{+}\left(D^{2} u\right)+u^{p}=0
$$

which change infinitely many times its concavity. These solutions are not present in the case of the Laplacian. These solutions appear since the system tries to compensate the fact that $\lambda<\Lambda$.

\section{Coffman-Kolodner analysis}

In this section we study the solutions obtained near a fast decaying solution, the idea is to vary $p$ in order to classify them. We differentiate the solution of (2.1)-(2.2) with respect to $p$, keeping the initial condition fixed. The resulting function $\varphi$ has valuable information on the solutions near the fast decaying one.

This idea was introduced by Coffman and Kolodner in studying uniqueness questions for semilinear equations. They differentiate with respect to the initial condition though.

By analyzing the function $\varphi$ we will prove in this section the following two propositions, that are crucial in the proof of our main results.

Proposition 4.1. - If $p^{*} \in \mathcal{F}$, then for $p<p^{*}$ close to $p^{*}$ we have $p \in \mathcal{C}$.

Proposition 4.2. - If $p^{*} \in \mathcal{F}$, then for $p>p^{*}$ close to $p^{*}$ we have $p \in \mathcal{S} \cup \mathcal{P}$.

For the proof of these propositions we need some preliminary lemmas. Since in our analysis $\gamma$ is kept fixed, so we do not make explicit mention of it. Its value will be chosen later.

Let $p^{*} \in \mathcal{F}$ and let $u\left(r, p^{*}\right)$ be the solution of (2.1)-(2.2). In view of the results in [6], in order to prove Theorems 1.1 and 1.2 we may assume that $p>\widetilde{N} /(\widetilde{N}-2)$. Since $p^{*} \in \mathcal{F}$ we have that $u$ changes its concavity only once, that is, there is the unique point $r_{0}=r_{0}\left(p^{*}\right)$ such that $u^{\prime \prime}\left(r_{0}\right)=0$. Moreover, the equation $u^{\prime \prime}\left(r_{0}\right)=0$ defines $r_{0}=r_{0}(p)$ as a $C^{1}$ function of $p$, in a neighborhood of $p^{*}$. These results are consequence of Lemma 3.1 and what follows.

LEMMA 4.1. $-r_{0}$ is a $C^{1}$ function of $p$, for $p$ near $p^{*}$. 
Proof. - The condition defining $r_{0}$ is $u^{\prime \prime}(p, r)=0$, that is

$$
\frac{\lambda(N-1)}{r} u^{\prime}(p, r)+u^{p}(p, r)=0 .
$$

In order to define $r_{0}$ as a function of $p$ we use the Implicit Function Theorem. It is enough to prove that

$$
S(r)=\left[\frac{-\lambda(N-1)}{r^{2}}+p u^{p-1}(p, r)\right] u^{\prime}(p, r)
$$

does not vanish at $r_{0}$. Using the Emden-Fowler transformation we see that this is equivalent to prove that

$$
S\left(\mathrm{e}^{t}\right)=p \mathrm{e}^{-2 t}\left(x^{p-1}-\frac{\lambda(N-1)}{p}\right)
$$

does not vanish at the crossing point. But we saw in the proof of Lemma 3.1 that at the crossing point $x>x_{1}$, where $x_{1}^{p-1}=\lambda(N-1) / p$. We complete the proof just observing that $x_{1}$ is the only point where $S$ vanishes.

Since the function $r_{0}(p)$ is a differentiable function, we can differentiate $u$ and $u^{\prime}$ with respect to $p$. We define $\varphi(r, p)=\partial u(r, p) / \partial p$ and we prove

LEMma 4.2. - The function $\varphi(\cdot, p)$ is a $C^{1}$ function in $\mathbb{R}^{+}$, for $p$ near $p^{*}$. Moreover, it satisfies the equations

$$
\lambda \varphi^{\prime \prime}+\frac{\lambda(N-1)}{r} \varphi^{\prime}+p u^{p-1} \varphi+u^{p} \log u=0 \quad \text { if } r<r_{0},
$$

and

$$
\Lambda \varphi^{\prime \prime}+\frac{\lambda(N-1)}{r} \varphi^{\prime}+p u^{p-1} \varphi+u^{p} \log u=0 \quad \text { if } r>r_{0} .
$$

Proof. - The function $u$ satisfies the equation

$$
\lambda u^{\prime \prime}+\frac{\lambda(N-1)}{r} u^{\prime}+u^{p}=0 \quad \text { if } r<r_{0}(p),
$$

so that, a direct computation shows that $\varphi$ satisfies (4.2), with initial conditions $\varphi(0)=0$ and $\varphi^{\prime}(0)=0$. Next we define the function $v(s, p)$ as the unique solution of the initial value problem

$$
\Lambda v^{\prime \prime}+\frac{\lambda(N-1)}{s+r_{0}(p)} v^{\prime}+v^{p}=0
$$

with initial conditions

$$
v(0)=u\left(r_{0}(p)^{-}, p\right) \quad \text { and } \quad v^{\prime}(0)=u^{\prime}\left(r_{0}(p)^{-}, p\right) .
$$


Then we see that $v$ is differentiable with respect to $p$ and $u(r, p)=v\left(r-r_{0}(p), p\right)$ for all $r \geqslant r_{0}(p)$. From here we find that $u(r, p)$ is also differentiable with respect to $p$.

Differentiating we have that for all $r>r_{0}(p)$

$$
\frac{\partial u}{\partial p}(r, p)=-v^{\prime}\left(r-r_{0}(p), p\right) r_{0}^{\prime}(p)+\frac{\partial v}{\partial p}\left(r-r_{0}(p), p\right),
$$

from where we obtain

$$
\frac{\partial u}{\partial p}\left(r_{0}(p)^{+}, p\right)=-v^{\prime}(0, p) r_{0}^{\prime}(p)+\frac{\partial v}{\partial p}(0, p) .
$$

If we consider the initial conditions (4.5) we finally find that

$$
\frac{\partial u}{\partial p}\left(r_{0}(p)^{+}, p\right)=\frac{\partial u}{\partial p}\left(r_{0}(p)^{-}, p\right) .
$$

In a similar way we obtain

$$
\frac{\partial u^{\prime}}{\partial p}\left(r_{0}(p)^{+}, p\right)=\frac{\partial u^{\prime}}{\partial p}\left(r_{0}(p)^{-}, p\right) .
$$

This last two equalities prove that $\varphi$ is of class $C^{1}$.

In the discussion to follow we will keep $p=p^{*}$ fixed. Then, for notational convenience, we will write $p$ instead of $p^{*}$. In the proof of Proposition 4.1 we will come back to the regular notation.

Now we fix the constant $\gamma>0$, the initial condition in (2.1)-(2.2), in such a way that $x^{\prime}(T)=0$ implies $u\left(e^{T}\right)=1$. This is possible since a change in $\gamma$ implies time translation in the dynamical system. The next lemma provides two identities that are very important in the sequel. These type of identities where introduced in [15], for a related problem.

Lemma 4.3. - Let $u(r, p)$ and $\varphi(r)$ as above. Then the following identities hold:

$$
\begin{aligned}
& \left\{r^{N-1}\left[(r u)^{\prime \prime} \varphi-(r u)^{\prime} \varphi^{\prime}\right]\right\}^{\prime}=\frac{r^{N-1}}{\lambda}\left[(p-3) u^{p} \varphi+r u^{\prime} u^{p} \log u+u^{p+1} \log u\right], \\
& \left\{r^{N-1}\left(u^{\prime} \varphi-u \varphi^{\prime}\right)\right\}^{\prime}=\frac{r^{N-1}}{\lambda}\left[(p-1) u^{p} \varphi+u^{p+1} \log u\right], \quad \text { for } r \geqslant r_{0},
\end{aligned}
$$

and

$$
\begin{aligned}
& \left\{r^{\widetilde{N}-1}\left[(r u)^{\prime \prime} \varphi-(r u)^{\prime} \varphi^{\prime}\right]\right\}^{\prime}=\frac{r^{\widetilde{N}-1}}{\Lambda}\left[(p-3) u^{p} \varphi+r u^{\prime} u^{p} \log u+u^{p+1} \log u\right], \\
& \left\{r^{\widetilde{N}-1}\left(u^{\prime} \varphi-u \varphi^{\prime}\right)\right\}^{\prime}=\frac{r^{\widetilde{N}-1}}{\Lambda}\left[(p-1) u^{p} \varphi+u^{p+1} \log u\right] \quad \text { and } r>r_{0} .
\end{aligned}
$$

Proof. - The proof is obtained by a routine calculation, starting from the equations satisfied by $\varphi$ and $u$. We omit the details.

The next lemma is a key step in our arguments. 
LEMMA 4.4. - It is not possible to have simultaneously that

$$
\lim _{r \rightarrow \infty} \varphi(r)=c_{1} \leqslant 0 \quad \text { and } \quad \lim _{r \rightarrow \infty} r \varphi^{\prime}(r)=0 .
$$

Proof. - Since $u$ is a fast decaying solution, there exists a $C>0$ such that

$$
\lim _{r \rightarrow \infty} u(r) r^{\widetilde{N}-2}=C \quad \text { and } \quad \lim _{r \rightarrow \infty} u^{\prime}(r) r^{\widetilde{N}-1}=(2-\tilde{N}) C
$$

Then

$$
\lim _{r \rightarrow \infty} r^{\widetilde{N}-1}\left(u^{\prime} \varphi-u \varphi^{\prime}\right)=(2-\tilde{N}) C c_{1} .
$$

On the other hand, using the equation for $u$ we find

$$
r^{\widetilde{N}-1}\left[(r u)^{\prime \prime} \varphi-(r u)^{\prime} \varphi^{\prime}\right]=r^{\widetilde{N}-1}\left[\left((3-\widetilde{N}) u^{\prime}-r u^{p}\right) \varphi-\left(u+r u^{\prime}\right) \varphi^{\prime}\right] .
$$

From here, and the fact that $p>\widetilde{N} /(\widetilde{N}-2)$, we obtain

$$
\lim _{r \rightarrow \infty} r^{\widetilde{N}-1}\left[(r u)^{\prime \prime} \varphi-(r u)^{\prime} \varphi^{\prime}\right]=(2-\widetilde{N})(3-\widetilde{N}) C c_{1}
$$

Now we integrate identities (4.7), (4.9) and (4.6), (4.8) and we use the limits (4.11) and (4.12) to find

$$
\begin{aligned}
& \int_{0}^{r_{0}} \frac{r^{N-1}}{\lambda}\left[(p-1) u^{p} \varphi+u^{p+1} \log u\right]+\beta \int_{r_{0}}^{\infty} \frac{r^{\widetilde{N}-1}}{\Lambda}\left[(p-1) u^{p} \varphi+u^{p+1} \log u\right] \\
& \quad=(2-\tilde{N}) C c_{1}
\end{aligned}
$$

and

$$
\begin{aligned}
& \int_{0}^{r_{0}} \frac{r^{N-1}}{\lambda}\left[(p-3) u^{p} \varphi+r u^{\prime} u^{p} \log u+u^{p+1} \log u\right] \\
& \quad+\beta \int_{r_{0}}^{\infty} \frac{r^{\widetilde{N}-1}}{\Lambda}\left[(p-3) u^{p} \varphi+r u^{\prime} u^{p} \log u+u^{p+1} \log u\right] \\
& \quad=(2-\widetilde{N})(3-\widetilde{N}) C c_{1},
\end{aligned}
$$

where $\beta=r_{0}^{N-\widetilde{N}}$. If we multiply the first integral by $(p-3) /(p-1)$ and subtract the second one we get

$$
\begin{aligned}
& \int_{0}^{r_{0}} \frac{r^{N-1}}{\lambda}\left[\left(\alpha u+r u^{\prime}\right) u^{p} \log u\right]+\beta \int_{r_{0}}^{\infty} \frac{r^{\widetilde{N}-1}}{\Lambda}\left[\left(\alpha u+r u^{\prime}\right) u^{p} \log u\right] \\
& \quad=\left(3-\tilde{N}-\frac{p-3}{p-1}\right)(2-\tilde{N}) C c_{1} .
\end{aligned}
$$


We notice that $x^{\prime}(t)=r^{\alpha}\left(\alpha u+r u^{\prime}\right)$, then $\alpha u+r u^{\prime}$ change the sign when $x^{\prime}$ does. But we have chosen $\gamma$ so that $\log u$ change sign when $x^{\prime}$ does. Thus $\left(\alpha u+r u^{\prime}\right) u^{p} \log u>0$, for all $r \geqslant 0$. On the other hand, since $p>\widetilde{N} /(\widetilde{N}-2)$ we have that the right-hand side in (4.13) is negative or zero, providing a contradiction.

Continuing with our analysis we define the function

$$
w=w_{\theta}(r)=r^{\theta} u(r, p),
$$

for $\theta>0$ chosen so that $\theta=(\widetilde{N}-1) / 2$ if $\widetilde{N}>3$ and $\theta=(\widetilde{N}-2) / 2$ if $2<\widetilde{N} \leqslant 3$. This function was introduced by Erbe and Tang in [8], for a related problem. The function $w$ satisfies the equation

$$
w^{\prime \prime}+\frac{(\widetilde{N}-1-2 \theta)}{r} w^{\prime}+\frac{\theta(\theta+2-\widetilde{N})}{r^{2}} w+r^{\theta} \frac{u^{p}}{\Lambda}=0 \quad \text { if } r>r_{0} .
$$

Next we define

$$
y(r)=\frac{\partial w(r)}{\partial p}=r^{\theta} \varphi
$$

When $\widetilde{N}>3$, the function $y$ satisfies the equation

$$
y^{\prime \prime}+\left(\frac{(\widetilde{N}-1)(3-\tilde{N})}{4 r^{2}}+\frac{p u^{p-1}}{\Lambda}\right) y+r^{\theta} \frac{u^{p}}{\Lambda} \log u=0 \quad \text { if } r>r_{0} .
$$

Since $u$ is a fast decaying solution we have (4.10), then we find that

$$
\lim _{r \rightarrow \infty} r^{(\widetilde{N}-2)(p-1)} u^{p-1}(r)=C^{p-1} .
$$

But, since $p>\widetilde{N} /(\widetilde{N}-2)$, we have that $(\widetilde{N}-2)(p-1)>2$. Thus the coefficient in the second term of (4.15) is negative for $r$ large.

When $2<\widetilde{N} \leqslant 3$, then $y$ satisfies the equation

$$
y^{\prime \prime}+\frac{y^{\prime}}{r}+\left(\frac{-(\widetilde{N}-2)^{2}}{4 r^{2}}+\frac{p u^{p-1}}{\Lambda}\right) y+r^{\theta} \frac{u^{p}}{\Lambda} \log u=0 \quad \text { if } r>r_{0} .
$$

Since, again we have (4.16), the coefficient of the third term in (4.17) is also negative for $r$ large.

Now we can prove the following lemma on the asymptotic behavior of $y$.

LEMMA 4.5. - The function y defined above satisfies $y(r)>0$ for $r$ large.

Proof. - Suppose, for contradiction, that there exists a $\bar{r}$ large such that $y(\bar{r})<0$, then we have the following two possibilities:

(a) $y(r)<0$ for all $r>\bar{r}$ or (b) there exists $r^{*}>\bar{r}$ such that $y\left(r^{*}\right)=0$ and $y^{\prime}\left(r^{*}\right) \geqslant 0$.

In case (a) we have that $\varphi(r)<0$ for all $r>\bar{r}$. From (4.9) we have then that for $r$ large

$$
\left\{r^{\widetilde{N}-1}\left(u^{\prime} \varphi-u \varphi^{\prime}\right)\right\}^{\prime}<0 \text { and } \quad\left\{r^{\widetilde{N}-1} \varphi^{\prime}\right\}^{\prime}>0 .
$$


Again there are two possibilities:

(i) There exists $\tilde{r}>\bar{r}$ such that $u^{\prime}(\tilde{r}) \varphi(\tilde{r})-u(\tilde{r}) \varphi^{\prime}(\tilde{r})<0$ or

(ii) $u^{\prime}(r) \varphi(r)-u(r) \varphi^{\prime}(r)>0$ for all $r \geqslant \bar{r}$.

If (i) is true, from (4.18) we have $u^{\prime}(r) \varphi(r)-u(r) \varphi^{\prime}(r)<0$ for all $r \geqslant \tilde{r}$, from where it follows that the function $u / \varphi$ is strictly decreasing for all $r \geqslant \tilde{r}$. Thus there is a number $c_{\infty}$, possibly $-\infty$, such that

$$
\lim _{r \rightarrow \infty} \frac{u(r) r^{\widetilde{N}-2}}{\varphi(r) r^{\widetilde{N}-2}}=c_{\infty},
$$

and then $\lim _{r \rightarrow \infty} \varphi r^{\widetilde{N}-2}=C / c_{\infty} \leqslant 0$, where $C$ is given in (4.10). On the other hand from (4.3) we have $\left.\left\{r^{\widetilde{N}-1} \varphi^{\prime}\right)\right\}^{\prime}>0$ for $r$ large, then there is a positive constant $c_{1}$, possibly $+\infty$, so that

$$
\lim _{r \rightarrow \infty} \varphi^{\prime} r^{\widetilde{N}-1}=c_{1}
$$

Hence by the L'Hospital's rule we get

$$
\lim _{r \rightarrow \infty} \varphi^{\prime} r^{\widetilde{N}-1}=(2-\widetilde{N}) \lim _{r \rightarrow \infty} \varphi r^{\widetilde{N}-2}=(2-\widetilde{N}) \frac{C}{c_{\infty}} .
$$

From here we obtain that $\varphi(r) \rightarrow 0$ and $r \varphi^{\prime}(r) \rightarrow 0$ as $r \rightarrow \infty$, contradicting Lemma 4.4.

If (ii) is true, we have

$$
u^{\prime}(r) \varphi(r)-u(r) \varphi^{\prime}(r)>0 \quad \text { for all } r \geqslant \tilde{r} .
$$

From (4.18) there exists $c_{2} \in(-\infty,+\infty]$ such that $\lim _{r \rightarrow \infty} \varphi^{\prime}(r) r^{\tilde{N}-1}=c_{2}$.

In case $c_{2} \leqslant 0$ we have $\varphi^{\prime}(r)<0$ for all $r$ large, consequently there exists $c_{1} \in$ $[-\infty, 0)$ such that $\lim _{r \rightarrow \infty} \varphi(r)=c_{1}$. We claim that $c_{1}$ is finite. In fact, we first observe that, since $\varphi^{\prime}(r) r^{\widetilde{N}-1}=r^{\widetilde{N}-2}\left(r \varphi^{\prime}(r)\right)$ converges to a finite limit, we have necessarily that $\lim _{r \rightarrow \infty} r \varphi^{\prime}(r)=0$. Then from (4.18) and (4.19) we find a finite constant $c \geqslant 0$ such that

$$
\lim _{r \rightarrow \infty} r^{\widetilde{N}-1}\left(u^{\prime}(r) \varphi(r)-u(r) \varphi^{\prime}(r)\right)=c,
$$

from where it follows that $c_{1}$ is finite. Thus we get a contradiction with Lemma 4.4.

In case $c_{2}>0$ then $\varphi^{\prime}(r)>0$ for all $r$ large, so that there exists a constant $c_{1} \in$ $(-\infty, 0]$ such that $\lim _{r \rightarrow \infty} \varphi(r)=c_{1}$.

Again we have (4.20) from where we find a non-negative constant $c_{3}$ such that $\lim _{r \rightarrow \infty} r \varphi^{\prime}(r)=c_{3}$. If $c_{3}>0$ then integrating this last limit we conclude that $\varphi$ is unbounded, which is impossible. Thus we again contradict Lemma 4.4.

In the second case (b), if $\widetilde{N}>3$ we have that $y(r)>0$ for all $r>r^{*}$ that is because (4.15) implies $y^{\prime \prime}(t)>0$ if $y(t) \geqslant 0$.

In case $2<\widetilde{N} \leqslant 3$, then there exists $r_{0}>r^{*}$ such that $y\left(r_{0}\right)>0$. In fact, the contrary would imply that $r^{*}$ is a local maximum point, contradicting the fact that $y^{\prime \prime}\left(r^{*}\right)>0$, 
as follows from (4.17). Now assuming $y\left(r_{0}\right)>0$, we see that $y(r)>0$ for all $r>r^{*}$, because the contrary would imply that $y$ has a local maximum point in $r_{1} \in\left(r^{*}, \infty\right)$ contradicting again that $y^{\prime \prime}\left(r_{1}\right)>0$, as follows again from (4.17).

COROLLARY 4.1. - The function y defined above, satisfies $y^{\prime}(r)>0$ for $r$ large.

Proof. - From Lemma 4.5 we have $y(r)>0$ for $r$ large, so in the case $\widetilde{N}>3$, by (4.15) we see that $y^{\prime \prime}(r)>0$ for $r$ large, hence $y$ is monotone. Thus we can define

$$
y_{\infty}=\lim _{r \rightarrow \infty} y(r) .
$$

If $0 \leqslant y_{\infty}<\infty$ then $\varphi(r) \rightarrow 0$ and $r \varphi^{\prime}(r) \rightarrow 0$ as $r \rightarrow \infty$. But this contradicts Lemma 4.4. Hence $y_{\infty}=\infty$, and then $y^{\prime}(r)>0$ for $r$ large.

In the case $2<\widetilde{N} \leqslant 3$, by (4.17) we have $\left\{r y^{\prime}\right\}^{\prime}(r)>0$. Consequently $r y^{\prime}$ is increasing, and then there exists $l \in(-\infty,+\infty]$ such that

$$
\lim _{r \rightarrow \infty} r y^{\prime}(r)=l \text {. }
$$

If $l<\infty$ we have

$$
\lim _{r \rightarrow \infty}\left(\frac{\widetilde{N}-2}{2}\right) r^{(\widetilde{N} 2) / 2} \varphi(r)+r^{(\widetilde{N}) / 2} \varphi^{\prime}(r)=l
$$

Then $\varphi(r) \rightarrow 0$ and $r \varphi^{\prime}(r) \rightarrow 0$ as $r \rightarrow \infty$, but this contradicts Lemma 4.4. Hence $l=\infty$ and then $y^{\prime}(r)>0$ for $r$ large.

Now we are prepared for proving Proposition 4.1. From now on we come back to our notation $p^{*} \in \mathcal{F}$.

Proof of Proposition 4.1. - Let $p^{*} \in \mathcal{F}$ and $p<p^{*}$ sufficiently close to $p^{*}$. Here, and in what follows, we assume that $u(0, p)=u\left(0, p^{*}\right)=\gamma$, where $\gamma$ was chosen before.

Suppose first that $p \in \mathcal{F}$ and $\widetilde{N}>3$. Let us define

$$
w(r)=r^{(\widetilde{N}-1) / 2} u(r, p), \quad w_{*}(r)=r^{(\widetilde{N}-1) / 2} u\left(r, p^{*}\right)
$$

and $v=w_{*}-w$. We see that for $r$ large $v$ satisfies the equation

$$
v^{\prime \prime}(r)+\frac{(\widetilde{N}-1)(3-\widetilde{N})}{4 r^{2}} v(r)+r^{(\widetilde{N}-1) / 2} \frac{\left(u\left(r, p^{*}\right)^{p^{*}}-u(r, p)^{p}\right)}{\Lambda}=0 .
$$

By the mean value theorem we have

$$
u\left(r, p^{*}\right)^{p^{*}}-u(r, p)^{p}=p^{*}(\xi(r))^{p^{*}-1}\left(u\left(r, p^{*}\right)-u(r, p)\right)+u(r, p)^{p^{*}}-u(r, p)^{p},
$$

where

$$
\xi(r) \in\left(\min \left\{u\left(r, p^{*}\right), u(r, p)\right\}, \max \left\{u\left(r, p^{*}\right), u(r, p)\right\}\right) .
$$


Next we use continuity of the solution of (2.1)-(2.2) with respect to the parameter $p$ and the fact that $u^{\prime}(r, p)<0$ for all $r>0$, to find $\bar{r}$ and $\varepsilon>0$ such that $u(r, p)<1$, for all $r \geqslant \bar{r}$ and for all $p \in\left(p^{*}-\varepsilon, p^{*}\right)$. Then $v$ satisfies

$$
v^{\prime \prime}+\left(\frac{(\tilde{N}-1)(3-\tilde{N})}{4 r^{2}}+\frac{p^{*}(\xi(r))^{p^{*}-1}}{\Lambda}\right) v \geqslant 0 \quad \text { for all } r \geqslant \bar{r} .
$$

Using (4.16) and that $p>\widetilde{N} /(\widetilde{N}-2)$ we conclude the existence of $r^{*}$ such that

$$
\frac{(\tilde{N}-1)(3-\tilde{N})}{4 r^{2}}+\frac{p^{*}\left(u\left(r, p^{*}\right)\right)^{p^{*}-1}}{\Lambda}<0 \text { for all } r \geqslant r^{*} .
$$

On the other hand, by Lemma 4.5 and Corollary 4.1, there exists $\tilde{r}$ such that $y(\tilde{r})>0$ and $y^{\prime}(\tilde{r})>0$, for $\tilde{r}>\max \left\{r^{*}, \bar{r}\right\}$. Thus $v(\tilde{r})>0$ and $v^{\prime}(\tilde{r})>0$ for a fix $p \in\left(p^{*}-\varepsilon, p^{*}\right)$ close to $p^{*}$. But since $p \in \mathcal{F}$ and $\widetilde{N}>3$, we have $v(r) \rightarrow 0$ as $r \rightarrow \infty$. Thus $v$ has a positive maximum, let us say in $\hat{r}$. Since $v(\hat{r})>0$, we get $u(\hat{r}, p)<u\left(\hat{r}, p^{*}\right)$, hence $u\left(\hat{r}, p^{*}\right)>\xi(\hat{r})$. Thus, from (4.24) we have

$$
\frac{(\widetilde{N}-1)(3-\widetilde{N})}{4 \hat{r}^{2}}+\frac{p^{*}(\xi(\hat{r}))^{p^{*}-1}}{\Lambda}<0 .
$$

But then we get a contradiction from (4.23), and the fact that $\hat{r}$ is a maximum of $v$.

In case $p \in \mathcal{F}$ and $2<\widetilde{N} \leqslant 3$ we proceed slightly different. Define

$$
w(r)=r^{(\widetilde{N}-2) / 2} u(r, p), \quad w_{*}(r)=r^{(\widetilde{N}-2) / 2} u\left(r, p^{*}\right)
$$

and $v=w_{*}-w$. We see that for $r$ large, $v$ satisfies

$$
v^{\prime \prime}(r)+\frac{v^{\prime}(r)}{r}+\frac{-(\widetilde{N}-2)^{2}}{4 r^{2}} v(r)+r^{(\widetilde{N}-2) / 2} \frac{\left(u\left(r, p^{*}\right)^{p^{*}}-u(r, p)^{p}\right)}{\Lambda}=0 .
$$

Then we use an argument similar to that of $\widetilde{N}>3$, and we get a contradiction again.

Suppose next that $p \in \mathcal{P} \cup \mathcal{S}$. We will distinguish two cases: $\lambda \leqslant \Lambda$ and $\Lambda<\lambda$, corresponding to $\mathcal{M}^{+}$and $\mathcal{M}^{-}$, respectively.

In case $\lambda \leqslant \Lambda$ we observe that the solution $u=u(r, p)$ satisfies

$$
\Lambda u^{\prime \prime}+\lambda \frac{N-1}{r} u^{\prime}+u^{p} \leqslant 0, \quad \forall r>0 .
$$

We see then that the argument given for $p \in \mathcal{F}$ can be repeated step by step since the function $v$ satisfies, for $r$ large,

$$
v^{\prime \prime}(r)+\frac{(\widetilde{N}-1)(3-\widetilde{N})}{4 r^{2}} v(r)+r^{(\widetilde{N}-1) / 2} \frac{\left(u\left(r, p^{*}\right)^{p^{*}}-u(r, p)^{p}\right)}{\Lambda} \geqslant 0,
$$

which is all we need. 
In case $\Lambda<\lambda$ we need some extra work. Assume first that $N>3$ and define the function $\bar{w}^{*}(r)=r^{(N-1) / 2} u\left(r, p^{*}\right)$ and $\bar{y}$ as

$$
\bar{y}(r)=\frac{\partial \bar{w}^{*}(r)}{\partial p}=r^{(N-1) / 2} \varphi .
$$

Then the function $\bar{y}$ satisfies the equation

$$
\begin{aligned}
\bar{y}^{\prime \prime} & +(\widetilde{N}-N) \frac{\bar{y}^{\prime}}{r}+\left(\frac{(N-1)(N+3-2 \widetilde{N})}{4 r^{2}}+\frac{p^{*} u\left(r, p^{*}\right)^{p^{*}-1}}{\Lambda}\right) \bar{y} \\
& +r^{(N-1) / 2} \frac{u\left(r, p^{*}\right)^{p^{*}}}{\Lambda} \log u=0,
\end{aligned}
$$

if $r>r_{0}$. We claim that $\bar{y}$ and $\bar{y}^{\prime}$ are positive, for $r$ large enough. In fact, by Lemma 4.5 we know that for $r$ large the function $y$ is positive, then $\bar{y}$ is also positive. On the other hand, as in (4.24) and noticing that $\tilde{N}>N$, we can find $r^{*}$ such that

$$
\frac{(N-1)(N+3-2 \tilde{N})}{4 r^{2}}+\frac{p^{*} u\left(r, p^{*}\right)^{p^{*}-1}}{\Lambda}<0 \text { for all } r \geqslant r^{*} .
$$

Thus, from (4.26) we get that $\left(r^{\widetilde{N}-N} \bar{y}^{\prime}\right)^{\prime}$ is positive for large $r$ and so that $\lim _{r \rightarrow \infty} r^{\widetilde{N}-N} \bar{y}^{\prime}$ $=l$ exists. If $l$ is finite we have that

$$
\lim _{r \rightarrow \infty} r^{\widetilde{N}-(N+1) / 2} \varphi^{\prime}(r)+\frac{N-1}{2} r^{\widetilde{N}-(N+3) / 2} \varphi(r)=l,
$$

from where we see that $\lim _{r \rightarrow \infty} r \varphi^{\prime}(r)=\lim _{r \rightarrow \infty} \varphi(r)=0$, contradicting Lemma 4.4. We conclude so that $l=+\infty$ and then $\bar{y}^{\prime}(r)>0$, for $r$ large, completing the proof of our claim.

Next we define $\bar{w}(r)=r^{(N-1) / 2} u(r, p)$ and $v=\bar{w}^{*}-\bar{w}$. By the positivity of $\bar{y}$ and $\bar{y}^{\prime}$ and the growth of $\bar{w}^{*}$ and $\bar{w}$, we find that, for certain $p$ close to $p^{*}, v$ possesses a positive maximum at a point $\bar{r}$.

If $u$ is convex at $\bar{r}$ then $v$ satisfies the equation

$$
v^{\prime \prime}+(\tilde{N}-N) \frac{v^{\prime}}{r}+\left(\frac{(N-1)(N+3-2 \widetilde{N})}{4 r^{2}}\right) v+r^{(N-1) / 2} \frac{u\left(r, p^{*}\right)^{p^{*}}-u(r, p)^{p}}{\Lambda}=0,
$$

and we can repeat the argument as in the case $p \in \mathcal{F}$.

If $u$ is concave at $\bar{r}$, the point of maximum of $v$, then we use that $u^{*}=u\left(\cdot, p^{*}\right)$ satisfies

$$
\lambda\left(u^{*}\right)^{\prime \prime}+\lambda(N-1) \frac{\left(u^{*}\right)^{\prime}}{r}+\left(u^{*}\right)^{p^{*}} \geqslant 0
$$

to find that $v$ satisfies the inequality

$$
v^{\prime \prime}+\left(\frac{(N-1)(3-N)}{4 r^{2}}\right) v+r^{(N-1) / 2} \frac{u\left(r, p^{*}\right)^{p^{*}}-u(r, p)^{p}}{\lambda} \geqslant 0 .
$$


From here we can reach a contradiction again, as in the case $p \in \mathcal{F}$.

It is only left the case $N=3$, but this is treated proceeding as before, but defining $\bar{w}^{*}(r)=r^{(N-2) / 2} u\left(r, p^{*}\right)$ and $\bar{w}(r)=r^{(N-2) / 2} u(r, p)$. This finishes the proof of the proposition.

Now we can complete the proof of Proposition 4.2, saying that above $p^{*} \in \mathcal{F}$ solutions are slow (or pseudo-slow) decaying.

Proof of Proposition 4.2. - Let us assume that $p \in \mathcal{C}$ and $p>p^{*}$. We have that the function $u\left(r, p^{*}\right)$ is convex for $r>r_{0}$ and that if $p$ is close enough to $p^{*}$, the function $u(r, p)$ is also convex for $r \in\left(r_{0}+1, r_{1}\right)$, where $r_{1}$ is the first point where $u(r, p)=0$. We notice that $r_{1}$ goes to infinity if $p \rightarrow p^{*}$.

Then in the range $r \in\left(r_{0}+1, r_{1}\right)$ we can proceed as in the proof of Proposition 4.1 (in the case $p \in \mathcal{F}$ ). The difference is that in (4.23) the inequality is reversed and at $\tilde{r}$ we have $v(\tilde{r})<0$ and $v^{\prime}(\tilde{r})<0$. The contradiction comes from the fact that $v\left(r_{1}\right)>0$ and so $v$ must have a minimum in $\left(r_{0}+1, r_{1}\right)$.

To conclude we notice that if $p>p^{*}$ and $p \in \mathcal{F}$, then $p \notin \mathcal{F}$, because Proposition 4.1 would imply that $\left(p^{*}, p\right) \cap \mathcal{C} \neq \emptyset$, which is impossible as we just proved.

\section{Proof of Theorems 1.1 and 1.2}

In this section we complete the proof of Theorems 1.1 and 1.2. We also state and prove an existence theorem in the case of the ball, which appears as a direct consequence of our analysis.

Proof of Theorem 1.1. - According to Propositions 3.1, 3.2 and Remark 2.1, the sets $\mathcal{C}$ and $\mathcal{P} \backslash\{(\widetilde{N}+2) /(\widetilde{N}-2)\}$ are open in $(1, \infty)$ and their boundary points are in $\mathcal{F}$. The fact that $\mathcal{F}$ is a Singleton is a direct consequence of Propositions 4.1 and 4.2. The growth rate of its single element is obtained by analyzing the linearization of the dynamical system near $(0,0)$ in $R^{-}$.

The rest of the theorem follows directly from Proposition 3.3.

Proof of Theorem 1.2. - As in Theorem 1.1, the fact that $\mathcal{F}$ is a Singleton is a direct consequence of Propositions 4.1 and 4.2 and the openness of $\mathcal{C}$ and of $\mathcal{P} \cup \mathcal{S}$. The rest of the theorem follows directly from Propositions 3.1 and 3.2.

Finally our existence theorem for a ball.

THEOREM 5.1. - Let $R>0$ and $B=B(0, R)$ the ball of radius $R$ centered at the origin. Then the equation

$$
\begin{aligned}
& \mathcal{M}_{\lambda, \Lambda}^{ \pm}\left(D^{2} u\right)+u^{p}=0 \quad \text { in } B, \\
& u(x)=0, \quad x \in \partial B, \quad u>0 \quad \text { in } B,
\end{aligned}
$$

has a unique solution provided $1<p<p_{ \pm}^{*}$. 
Proof. - In the range of $p$ all solutions of the initial value problem (2.1) are crossing solutions. Then by choosing $\gamma$ appropriately we obtain a solution of (5.1), which is unique.

\section{REFERENCES}

[1] X. Cabré, L.A. Caffarelli, Fully Nonlinear Elliptic Equation, in: Colloquium Publication, Vol. 43, American Mathematical Society, 1995.

[2] L. Caffarelli, B. Gidas, J. Spruck, Asymptotic symmetry and local behavior of semilinear elliptic equations with critical Sobolev growth, Comm. Pure Appl. Math. 42 (3) (1989) 271-297.

[3] W. Chen, C. Li, Classification of solutions of some nonlinear elliptic equations, Duke Math. J. 3 (3) (1991) 615-622.

[4] C. Clemons, C. Jones, A geometric proof of Kwong-Mc Leod uniqueness result, SIAM J. Math. Anal. 24 (1993) 436-443.

[5] C. Coffman, Uniqueness of the ground state solution for $\Delta u-u+u^{3}=0$ and a variational characterization of other solutions, Arch. Rational Mech. Anal. 46 (1972) 81-95.

[6] A. Cutri, F. Leoni, On the Liouville property for fully nonlinear equations, Ann. Inst. H. Poincaré Analyse non lineaire 17 (2) (2000) 219-245.

[7] Y. Deng, D. Cao, Uniqueness of the positive solution for singular non-linear boundary value problems, Syst. Sci Math. Sci. 6 (1993) 25-31.

[8] L. Erbe, M. Tang, Structure of positive radial solutions of semilinear elliptic equation, J. Differential Equations 133 (1997) 179-202.

[9] B. Gidas, Symmetry and isolated singularitiesof positive solutions of nonlinear elliptic equations, in: Nonlinear Partial Differential Equations in Engineering and Applied Science (Proc. Conf., Univ. Rhode Island, Kingston, RI, 1979), in: Lecture Notes in Pure Appl. Math., Vol. 54, Dekker, New York, 1980, pp. 255-273.

[10] B. Gidas, J. Spruck, Global and local behavior of positive solutions of nonlinear elliptic equations, Comm. Pure Appl. Math. 34 (1981) 525-598.

[11] J. Hale, Ordinary Differential Equation, Wiley, New York, 1969.

[12] R. Kajikiya, Existence and asymptotic behavior of nodal solution for semilinear elliptic equation, J. Differential Equations 106 (1993) 238-256.

[13] I. Kolodner, The heavy rotating string - a nonlinear eigenvalue problem, Comm. Pure Appl. Math. 8 (1955) 395-408.

[14] M.K. Kwong, Uniqueness of positive solution of $\Delta u-u+u^{p}=0$ in $\mathbb{R}^{N}$, Arch. Rational Mech. Anal. 105 (1989) 243-266.

[15] M.K. Kwong, L. Zhang, Uniqueness of positive solution of $\Delta u+f(u)=0$ in an annulus, Differential Integral Equations 4 (1991) 583-596.

[16] W.M. Ni, R. Nussbaum, Uniqueness and nonuniqueness for positive radial solutions of $\Delta u+f(u, r)=0$, Comm. Pure Appl. Math. 38 (1985) 67-108.

[17] S.I. Pohozaev, Eigenfunctions of the equation $\Delta u+\lambda f(u)=0$, Soviet Math. 5 (1965) $1408-1411$. 